Case Report

\title{
Splenic undifferentiated high grade pleomorphic sarcoma of a small size with fatal tumor rupture
}

\author{
Amatya $\mathrm{BM}^{1,5}$, Sawabe $\mathrm{M}^{1}$, Arai $\mathrm{T}^{1}$, Kumakawa $\mathrm{T}^{2}$, Takubo $\mathrm{K}^{3}$, Kimula $\mathrm{Y}^{4}$, \\ Koike $\mathrm{M}^{5}$
}

${ }^{1}$ Departments of Pathology and Tokyo Metropolitan Geriatric Hospital, Tokyo, Japan

${ }^{2}$ Internal Medicine, Tokyo Metropolitan Geriatric Hospital, Tokyo, Japan

${ }^{3}$ Human Tissue Research Group, Tokyo Metropolitan Institute of Gerontology, Tokyo, Japan

${ }^{4}$ Department of Pathology, Ome Municipal General Hospital, Tokyo, Japan

${ }^{5}$ Department of Human Pathology, Tokyo Medical and Dental University, Graduate School, Tokyo, Japan

\section{Keywords: \\ Undifferentiated pleomorphic sarcoma; Malignant fibrous histiocytoma; Splenic neoplasms; Retroperitoneal hemorrhage}

\begin{abstract}
Primary undifferentiated pleomorphic sarcoma of the spleen is a rare and highly aggressive neoplasm that usually presents with splenomegaly, constitutional symptoms and frequent distant metastases. We report a case of 77-year old male patient with a past history of dissecting aortic aneurysm that developed acute hemorrhagic shock. Aneurysmal rupture was clinically suspected, but the postmortem examination revealed a $25 \mathrm{~mm}$-sized tumor in an atrophic spleen weighing 65 gram with massive retroperitoneal bleeding. Metastases were found in the right renal hilus, the right adrenal gland and femoral bone marrow. Histology of the tumor showed undifferentiated pleomorphic sarcoma. Tumor rupture with fatal bleeding and systemic metastases had occurred despite the small size of the tumor. Tumor size is not a reliable predictor of systemic metastasis or tumor rupture for splenic undifferentiated pleomorphic sarcoma.
\end{abstract}

\section{INTRODUCTION}

Undifferentiated high grade pleomorphic sarcoma is synonymous to pleomorphic undifferentiated malignant fibrous histiocytoma (MFH). It shows so-called MFHhistological pattern, and lacks evidences of any definable

\section{Correspondence:}

Dr. Motoji Sawabe, MD

Department of Pathology and Bioresource Center for Geriatric Research, Tokyo Metropolitan Geriatric Hospital

35-2 Sakae-cho, Itabashi, Tokyo 173-0015, Japan

Phone: +81-3-3964-1141, Fax: +81-3-3964-1982

E-mail: sawabe@tmig.or.jp lines of differentiation. The group of MFH-like sarcomas is the most common soft tissue sarcoma in patients over 40 years of age. It occurs most commonly in extremities (especially the lower limb) and less often trunk. ${ }^{1}$ When occurring in an extremity, it usually presents as a painless mass, enlarging over several months. The retroperitoneal MFH occasionally presents with constitutional symptoms such as malaise, anorexia and weight loss along with signs of increased intra-abdominal pressure. Meanwhile, the commonest malignant splenic neoplasms are leukemias and lymphomas, where the spleen is secondarily involved as part of systemic disease. Other types of primary malignant splenic 


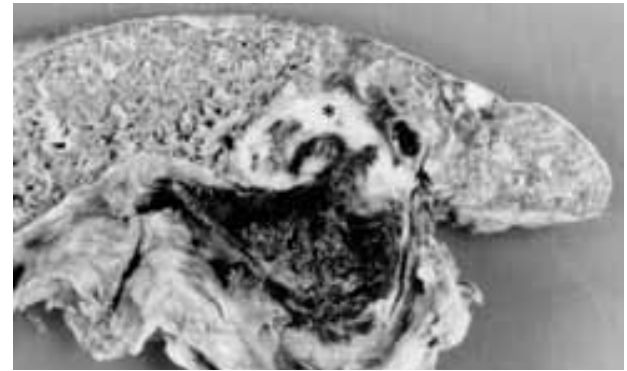

Figure 1: Cut surface of the spleen demonstrates a yellowish-white infiltrative tumor (asterisk) located at the hilum. Note tumor rupture and massive hemorrhage. Each segment of the scale represents $5 \mathrm{~mm}$.

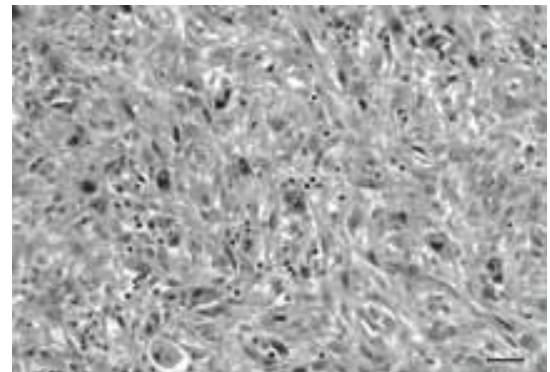

Figure 2: Histology of the splenic tumor shows a cellular neoplasm composed of atypical spindle cells with frequent mitoses and histiocyte-like cells with abundant cytoplasm. There are also multinucleated tumor giant cells and infiltrating small lymphocytes. (HE stain, X200).

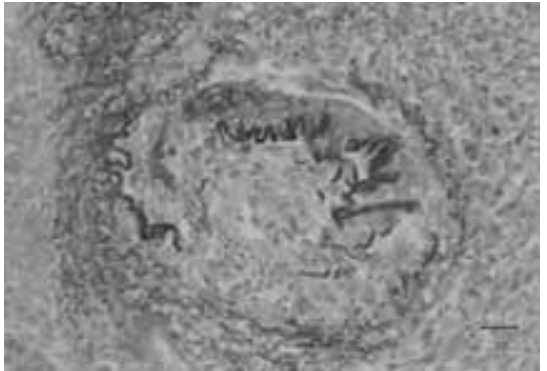

Figure 3: Invasive tumor cells destroy the splenic trabeculae and permeate intratrabecular arterioles and veins. (Elastic van-Gieson stain, X40) neoplasm are very rare; the majority being angiosarcomas and fibrosarcomas. ${ }^{2}$ Less than 20 cases of splenic MFH has been reported in English literatures so far. ${ }^{2-12}$ The review of 11 previously reported cases of splenic MFH showed it as a highly aggressive behaviour that usually presents with splenomegaly, constitutional symptoms, and frequent distant metastasis. ${ }^{7}$ Here, we report a case of a small splenic undifferentiated pleomorphic sarcoma which presented with fatal acute hemorrhagic shock. To our knowledge, tumor rupture with fatal bleeding is an unusual complication of this rare splenic neoplasm.

\section{CASE REPORT}

A 77 year-old severely emaciated male patient (body weight, $35 \mathrm{~kg}$ ) was admitted to the Tokyo Metropolitan Geriatric Hospital, Tokyo, Japan, after suffering from facial trauma due to fall. Personal history revealed that he was a non-smoker and never consumed alcohol. He was diagnosed with hypertension and angina pectoris at the age of 71 years and was under regular medications. Four years prior to the admission; thrombocytopenia was observed. Four months prior to admission, he developed a dissecting aortic aneurysm (DeBakey type IIIb) for which he was treated conservatively. He had experienced gradual loss of appetite, episodes of severe nasal bleeding and occasional melaena for one week prior to admission. On admission, there were ecchymoses around his left eye and left lip, due to facial injury. A pulsatile abdominal mass was present with an associated systolic murmur. There were multiple petechiae over the limbs. Blood count showed severe anemia (haemoglobin, $4.6 \mathrm{~g} / \mathrm{dL}$ ) and thrombocytopenia (platelet count, 45,000/cumm). An abdominal echogram noted an abdominal aortic aneurysm and a left renal cyst. There was no evidence of hepatosplenomegaly. An abdominal CT scan confirmed an unruptured aortic aneurysm. ${ }^{99 m} \mathrm{Tc}-\mathrm{HSA}$ (human serum albumin) scintigraphy detected a positive signal in the lower abdomen and in the left flank, consistent with internal bleeding. The clinical impression on admission was acute circulatory failure and chronic Disseminated Intravascular Coagulopathy (DIC). The patient was treated with haemostatic agents and was transfused with concentrated red cells, platelets, and fresh frozen plasma for his anemia and thrombocytopenia. Low-molecular-weight heparin was administered for DIC. Candida guilliermondii was isolated from a blood culture in the third week of admission. In the fourth week of admission he suddenly became severely hypotensive, with massive hematuria and worsening anemia. Rupture of the dissecting aneurysm was suspected. His general condition deteriorated and ultimately died of hemorrhagic shock. An autopsy was performed.

\section{Pathological findings}

The postmortem examination revealed massive retroperitoneal and intraperitoneal hemorrhage $(400 \mathrm{ml}$ pure blood) from a ruptured tumor at the splenic hilum. The tumor was white, fleshy, well-circumscribed, multilobated mass which measured $25 \mathrm{~mm}$ in diameter (fig. 1). The spleen weighed $65 \mathrm{gm}$. The tumor composed of a mixture of spindle cells with blunt-ended elongated nuclei, and histiocyte-like cells with large nuclei and abundant eosinophilic cytoplasm, arranged in a storiform pattern (fig. 2). There were some multinucleate giant cells and pleomorphic, undifferentiated cells scattered haphazardly in the hemorrhagic background. Some tumor cells showed erythrophagocytosis and contained hemosiderin pigment. There were frequent mitotic figures. Remnants of white pulp and some infiltrating small lymphocytes were observed within the tumor. Tumor cells occasionally destroyed splenic trabeculae and invaded trabecular arterioles and veins (fig.3). With this the tumor was diagnosed as undifferentiated pleomorphic sarcoma.

There was massive haemorrhage from the site of tumour rupture. Local tumor invasion into extrasplenic adipose tissue was also identified near the site of rupture. There was a $20 \mathrm{~mm}$-sized metastastic focus in right renal hilum that had permeated renal arterial branches at the hilum and embolized small distal branches, resulting in renal cortical infarcts. There were also metastases in the right adrenal gland and right femoral bone marrow. No lymphatic metastases were found. 
Immunohistochemical studies on paraffin-embedded sections showed the tumor cells were positive for vimentin and CD68 but negative for epithelial membrane antigen (EMA), S-100 protein, $\alpha$-smooth muscle actin, cytokeratin AE1+3, desmin, CD45, CD20, CD45RO, and HMB45. The majority of lymphocytes infiltrating the splenic tumor were CD45RO-positive $T$ cells. Ultrastructural study of formalin fixed tissue showed the tumor comprising of histiocyte-like cells with irregular nuclei and many cytoplasmic lysosomes.

The aorta showed severe atherosclerosis and there was an unruptured dissecting aneurysm, which extended from aortic arch to the level of origin of the renal arteries.

\section{DISCUSSION}

Less than 20 cases of splenic MFH have been reported in recent literature and the entity has been described as a rare and aggressive sarcoma which often shows systemic metastases at the time of clinical presentation..$^{2-12}$ Splenic MFH can present in various ways ranging from splenomegaly, abdominal pain, and ascites, to systemic manifestations including fever, cachexia, and prominent leukocytosis. Wick et $\mathrm{al}^{12}$ reported 3 cases of primary MFH of the spleen, 2 of which had presented with systemic manifestations of fever, weight loss, and malaise in addition to splenomegaly. ${ }^{12}$ Inflammatory MFH has some features distinct from other subtypes of MFH. It most often occurs in retroperitoneum rather than the extremities, and often presents with a systemic paraneoplastic syndrome, fever and leukocytosis, including neutrophilia or eosinophilia. ${ }^{13}$ The present case did not show any signs of systemic manifestation other than weight loss. A case of MFH arising in the descending aorta and manifesting as multiple thrombotic and tumor emboli to multiple organs, including the spinal cord and ribs, was also reported. ${ }^{14}$ This case is similar to the present case with respect to the presence of tumor embolization with resulting infarcts of the kidney.

Despite the small size of the splenic tumor in our case, it presented in a catastrophic manner and had systemic metastases. The Armed Forces Institute of Pathology third series fascicle on soft tissue tumors considers tumor size of less than $5 \mathrm{~cm}$ to be a good prognostic factor for MFH. ${ }^{15}$ According to the review of 11 cases of splenic MFH, the tumors were usually large and the spleens weighed 490 to $1870 \mathrm{~g}$ at the time of splenectomy. ${ }^{7}$ Recently one case of spontaneous rupture of a primary splenic MFH has been reported. ${ }^{3}$ This case had a ruptured splenic tumor measuring $6 \mathrm{~cm}$ in diameter which was larger than that of our case and died of metastatic diseases seven months after splenectomy.

\section{CONCLUSION}

The present case shows that even a small splenic undifferentiated pleomorphic sarcoma can rupture and metastasize, and the outcome cannot be predicted from the size of the primary tumor. The possibility of spontaneous rupture of an intra-abdominal sarcoma should be considered in the differential diagnosis of acute intra-abdominal bleeding.

\section{ACKNOWLEDGMENT}

The authors are indebted to the staff of the Department of Pathology, Tokyo Metropolitan Geriatric Hospital, for preparation of immunohistochemical slides.

\section{REFERENCES}

1. Fletcher CDM, van den Berg E, Molenaar WM. Pleomorphic malignant fibrous histiocytoma/Undifferentiated high grade pleomorphic sarcoma, In: Fletcher CDM, Unni K, Mertens F (eds). World Health Organization classification of tumours. Pathology and genetics of tumours of soft tissue and bone. IARC: Lyon; 2002. pp120-2.

2. Warnke RA, Weiss LM, Chan JKC, Cleary ML, Dorfman RF. Non-hematolymphoid tumors and tumor like lesions. In: Atlas of Tumor Pathology, Tumors of lymph nodes and spleen. Third series, Washington, DC: Armed Forces Institute of Pathology, 2001. pp502.

3. He L, Zhang H, Li X, Zhou J, Dong M. Primary malignant fibrous histiocytoma of spleen with spontaneous rupture: a case report and literature review. Med Oncol 2011;28:397-400.

4. Hashmi A, Podgaetz E, Richards ML. Laparoscopic resection of an undifferentiated pleomorphic splenic sarcoma. JSLS 2010;14:42630 .

5. Mantas D, Karidis N, Papachristodoulou A. Primary malignant fibrous histiocytoma of the spleen--an extremely rare entity. Acta Chir Belg 2010;110:558-60.

6. Ozaras R, Mert A, Avsar S et al. Malignant fibrous histiocytoma of the spleen. Intern Med 2003;42:1054.

7. Colovic N, Cemerikic-Martinovic V, Colovic R, Zogovic S. Primary malignant fibrous histiocytoma of the spleen and liver. Med Oncol 2001;18:293-7.

8. Mallipudi BV, Chawdhery MZ, Jeffery PJ. Primary malignant histiocytoma of spleen. Eur J Surg Oncol 1998;24:448-9.

9. Sieber SC, Lopez V, Rosai J, Buckley PJ. Primary tumor of the spleen with morphologic features of malignant fibrous histiocytoma. Immunohistochemical evidence for a macrophage origin. Am J Surg Pathol 1990;14:1061-70.

10. Jinno K, Moriwaki S. An autopsied case of malignant fibrous histiocytoma of the spleen. Gan No Rinso 1987;33:736-41.

11. Govoni E, Bazzocchi F, Pileri S, Martinelli G. Primary malignant fibrous histiocytoma of the spleen: an ultrastructural study. Histopathology 1982;6:351-61.

12. Wick MR, Scheithauer BW, Smith SL, Beart RW Jr. Primary nonlymphoreticular malignant neoplasms of the spleen. Am J Surg Pathol 1982;6:229-42.

13. Weiss SW, Goldblum JR. Malignant fibrohistiocytic tumors. In: Enzinger and Weiss's soft tissue tumors. 4th ed. Washington, DC: The C.V Mosby, 2001:pp539-68.

14. Riebeling V, Linné R, Bückmann FW, Markgraf R, Walther OE, Scholten T. Malignant fibrous histiocytoma of the aorta. Dtsch Med Wochenschr 1992;117:216-20.

15. Kempson RL, Fletcher CDM, Evans HL, Hendrickson MR, Sibley RK, editors. Atlas of Tumor Pathology, Tumors of the soft tissues. Third series. Armed Forces Institute of Pathology: Washington; 2001. pp159-86. 